\title{
Дорожная карта совершенствования деятельности проектов ДРВ на основе многопараметрических оценок
}

\author{
В.Н. Якимец ${ }^{1,2}$, И.И. Курочкин ${ }^{1}$ \\ ${ }^{1}$ Институт проблем передачи информации РАН, \\ ${ }^{2}$ Российская академия народного хозяйства и государственной службы \\ i aki mets@mail.ru, kurochkin@i itp.ru
}

\section{Аннотация}

Рассматривается методология развития проектов распределенных вычислений с участием добровольцев на основе применения результатов многопараметрических и индексных оценок. Такие оценки проектов позволяют выбрать стратегию совершенствования работы проекта добровольных распределенных вычислений упорядочить процесс построения соответствующей дорожной карты. Вводится понятие «целевой ориентир», с помощью которого команда проекта, учитывая его специфику и имеющиеся ресурсные возможности, по всем параметрам формирует вектор целевых значений, которые собирается достичь в течение заданного времени. Предложены четыре варианта выбора «целевых ориентиров», задающих направления дорожной карты развития проекта добровольных распределенных вычислений. Описываются результаты опроса сообщества добровольцев по многопараметрической оценке проектов за 2016-2018 годы. На примере проекта Gerasim@home показана реализация пути его совершенствования для выбранного варианта целевых ориентиров. Обсуждаются результаты изменений деятельности проекта.

Ключевые слова: распределенные вычисления, проект добровольных распределенных вычислений, многопараметрическая оценка проекта, индекс оценки проекта, целевые ориентиры, дорожная карта развития проекта

Библиографическая ссылка: Якимец В.Н., Курочкин И.И. Дорожная карта совершенствования деятельности проектов ДРВ на основе многопараметрических оценок // Информационное общество: образование, наука, культура и технологии будущего. Выпуск 3 (Труды XXII Международной объединенной научной конференции «Интернет и современное общество», IMS-2019, Санкт-Петербург, 19 22 июня 2019 г. Сборник научных трудов). - СПб: Университет ИТМО, 2019. C. 41 - 52. DOI: $10.17586 / 2587-8557-2019-3-41-52$

\section{1. Введение}

Использование грид-систем из персональных компьютеров (ГСПК) для решения научных вычислительных задач набрало популярность в самом конце XX века. При недостатке вычислительных мощностей многопроцессорных вычислительных систем для определенного типа задач, разделяемого по данным (bag of tasks) [1] стали использоваться различные распределенные вычислительные системы. Идея использования простаивающих ресурсов персональных компьютеров и других персональных устройств в научных вычислительных экспериментах стала особо популярна, как с точки зрения эффективного использования ресурсов, так и с точки зрения создания распределенных вычислительных систем на основе имеющихся вычислительных ресурсов. 
Появилось программное обеспечение по организации распределенных вычислительных систем, к примеру, Legion, HTCondor, Globus toolkit, Oracle Grid Engine, BOINC [2]. C помощью данного программного обеспечения можно было быстро и просто развертывать вычислительные грид-системы, в которых использовались вычислительные мощности как учебных и научных организаций, так и мощности добровольцев.

Грид-системы, развернутые для решения одной научной задачи или для проведения серии однотипных экспериментов, можно назвать проектами распределенных вычислений. Если при этом использовались вычислительные мощности добровольцев, то проектами добровольных распределенных вычислений (проекты ДРВ). Как правило, организаторами проектов ДРВ становились группы ученых, которые были заинтересованы, прежде всего, в проведении больших вычислительных экспериментов. Кроме привлечения вычислительных мощностей добровольцев, организаторы проектов ДРВ могли использовать проект ДРВ для популяризации, как своих научных исследований, так и науки в целом.

Сообщество добровольцев на данный момент составляет несколько миллионов человек со всего мира, а совокупная мощность уже подключенных вычислительных устройств к проектам ДРВ только на платформе BOINC сопоставима с самыми мощными суперкомпьютерами из рейтинга top500 [3]. Одним из крупнейших проектов на платформе BOINC является проект World Community Grid корпорации IBM, в котором участвуют более 700 тысяч пользователей и подключены более 5 миллионов вычислительных устройств (персональных компьютеров, серверов, смартфонов и др.) [4]. Проект является зонтичным [5] и объединяет несколько медицинских подпроектов по актуальным проблемам: поиск лекарства от рака, поиск лекарств от вируса Зика и др. Или, к примеру, первый успешный российский проект распределенных вычислений SAT@home для решения задач с помощью SAT-подхода [6]. В рамках научных проектов распределенных вычислений добровольцы не только предоставляют свои вычислительные ресурсы, но и обрабатывают изображения и другие полученные данные, ведут наблюдения за птицами, насекомыми и звездным небом. К примеру, проект по классификации различных типов галактик Zooniverse [7]. Или группа проектов орнитологической лаборатории Корнельского университета по наблюдению за птицами [8].

Использование добровольцев в научных проектах обозначается термином гражданская наука (citizen science) и является видом краудсорсинга (crowdsourcing) [9]. Как правило, для добровольцев даются достаточно простые задания: обработать фотографию по определенному алгоритму, найти отличия на двух похожих изображениях, найти и выделить на спутниковых снимках определенные объекты.

Взаимодействие с сообществом добровольцев при решении определенных научных задач позволяет увеличить масштаб проводимых исследований и освободить ученых от рутинной неквалифицированной работы. Улучшение взаимопонимания с отдельными добровольцами и сообществом в целом позволит повысить эффективность выполнения проекта [10]. Достичь взаимопонимания без обратной связи от сообщества добровольцев будет сложно, поэтому инструменты для формирования различных оценок необходимы для эффективного сопровождения проекта с использованием добровольцев.

В рамках задач прогнозирования, управления развитием разных областей знаний, технологий, продуктов и т.п. в последнее время получил широкое применение метод построения дорожных карт [11-17]. Чаще всего, его ассоциируют с применением методов прогнозирования, основанных на использовании концепции форсайта (от английского слова "foresight" (предвидение). Концепция форсайта - «это система методов стратегического управления, включая экспертные процедуры, позволяющие выявлять социально-экономические и научно-технические прорывы, которые способны оказать максимальное воздействие на экономику и общество в средне- и долго-срочной перспективах» [18]. 
Существует большое разнообразие определений понятия «Дорожная карта» и подходов к их построению. В [11] на странице 77 дорожной картой назван «документ, разработанный на основе обобщенного мнения экспертного сообщества и содержащий описание и визуальное представление глобальных и национальных вызовов, а также важнейших мероприятий, способных оказать существенное влияние на развитие рассматриваемой предметной области».

В работе [19] сказано, что дорожная карта, ничто иное как «расширенное видение будущего в выбранном направлении исследований, состоящее из коллективных знаний и представлений ведущих ученых-новаторов в этой области».

Имея в виду эти и другие сходные определения, рассмотрим вариант построения дорожной карты с учетом специфики объекта наших исследований - проектов добровольных распределенных вычислений (ДРВ). Под дорожной картой мы будем понимать выбранный вариант развития (или совершенствования) деятельности проекта ДРВ, отвечающий групповому мнению представителей распределенной команды участников проекта, содержащий описание и визуальное представление желаемого состояния проекта ДРВ с учетом возможностей и ресурсной обеспеченности команды.

Применительно к проектам ДРВ дорожная карта представляет собой развернутое видение будущего в специфичном для этого проекта содержательном направлении, и такое видение основывается на коллективных знаниях и оценках ученых и специалистов, работающих в этой сфере деятельности.

В широкий обиход вошло понятие метода дорожного картирования $[12,14,15,16]$. В [12] заявлено о развитии системного подхода к разработке дорожных карт для российских отраслей и компаний. Автор [15] претендует на создание унифицированной методики дорожного картирования. В работе [16] изложена технология дорожного картирования.

Ранее, для множества изучаемых проектов ДРВ была разработана единая методология построения многопараметрического «портрета», получаемого в результате обработки индивидуальных оценок специалистами текущего состояния проектов по заранее выбранным шкалам $[20,21]$. На основе усредненных групповых оценок каждого проекта ДРВ были выявлены различия многопараметрических «портретов» [21]. У одних проектов выявилась группа параметров с групповыми оценками ниже средних, у других - наличие подмножества параметров с относительно высокими оценками, у третьих преобладают средние оценки.

Естественным образом встала задача, что и как можно предложить сделать командам проектов, чтобы улучшить «портреты» проектов, исходя из возможностей и ограничений команд.

\section{2. Грид-системы из персональных компьютеров}

Организаторы проектов ДРВ помимо решения научных задач накладывают на себя дополнительные обязанности:

— разворачивание и сопровождение проекта ДРВ;

- увеличение вычислительной способности проекта ДРВ техническими методами (тонкая настройка ГСПК);

- увеличение вычислительной способности проекта ДРВ с помощью привлечения ресурсов добровольцев;

— удержание вычислительных ресурсов добровольцев в проекте ДРВ.

Если команда организаторов проекта ДРВ состоит из одного или нескольких человек, то решение этих проблем осуществляется по остаточному принципу. Но потребность в методике определения наиболее значимых направлений развития проекта ДРВ очень высока. 


\section{3. Дорожная карта для проекта ДРВ}

Разработка дорожной карты в отношении развития нашего объекта необходима и обоснована, если руководство и команда проекта ДРВ, изучив многопараметрические оценки состояния проекта, задумывается о возможности конкурентного развития и стремится рассмотреть имеющиеся пути формирования стратегического плана внесения преобразований в деятельность ДРВ-проекта.

Известно, что грамотно оформленная ДК должна содержать следующие важные компоненты:

— цель проекта;

- перечень важных задач (мероприятий) с определением лиц, ответственных за их реализацию;

- значимые требования к результатам мероприятий (работ), за выполнение которых несут ответственность определенные члены команды ДРВ-проекта;

- оценку временных затрат на выполнение каждого мероприятия;

- возможные варианты различных путей реализации проекта и контрольные точки на каждом этапе его реализации.

В нашем случае цель проекта состоит в том, чтобы сформировать набор возможных стратегий развития отдельных ДРВ-проектов, опираясь на многопараметрические оценки их состояния, и выбрать приемлемый вариант стратегии, улучшающей состояние ДРВпроекта с учетом возможностей и ограничений команды.

Мы не будем заниматься здесь детальным рассмотрением работы по компонентам 2-4 ДК, а сосредоточимся на компоненте 5 .

Ниже предлагается несколько альтернативных вариантов построения дорожных карт по улучшению состояния ДРВ-проектов, ориентированных на достижение целевых ориентиров по заранее определенному командой проекта подмножеству параметров

Введем понятие целевого ориентира (далее ЦО) при создании дорожной карты. ЦО это желаемое и достижимое значение отдельного параметра ДРВ-проекта, относительно величины которого у команды имеется консенсус (в форме квалифицированного или простого большинства). Значение ЦО по каждому согласованному параметру выбирается в интервале [0 - 1] и помечается верхней звездочкой. Например, $0.75^{*}$.

Предлагаются 4 варианта определения ЦО, задающие формирование возможных стратегий улучшения деятельности ДРВ-проекта:

1. Неравнозначные ЦО для всех параметров: ориентация на достижение командой проекта вектора неравнозначных, но желаемых и достижимых ЦО по всем параметрам с учетом возможностей команды.

2. Задание ЦО для выбранного подмножества параметров: ориентация на достижение командой проекта вектора желаемых и достижимых ЦО по выбранным параметрам с учетом специфики проекта.

3. ЦО для выбранного подмножества приоритетных параметров (имеющих высокие значения коэффициентов значимости): ориентация команды на повышение значений подмножества наиболее значимых параметров оценки ДРВ-проектов.

4. ЦО для выбранного подмножества параметров, по которым проектом были получены низкие оценки.

Построение дорожной карты для каждого ДРВ-проекта с использованием названных вариантов состоит из следующих этапов:

1. Команда проекта выбирает один или все 4 подхода.

2. Для каждого выбранного подхода устанавливаются ЦО по заданному числу параметров. Применяется правило консенсуса или большинства. Таким образом, формируется вектор ЦО $=\{Ц О(1), Ц О(2), \ldots, Ц \mathrm{O}(\mathrm{m})\}$. Здесь $\mathrm{m}-$ число параметров, выбранных командой проекта в рамках подходов В, C и D. В случае варианта А вектор включает все $\mathrm{n}$ параметров. 
3. Производится расчет значений ЯК-индекса с использованием вектора ЦО.

4. После расчета значений ЯК-индекса по значениям ЦО для каждого из 4 подходов производится их сравнение и с учетом возможностей команды принимается решение о выборе приемлемой стратегии реализации улучшения деятельности ДРВ-проекта.

5. Формируется набор задач и действий, которые должны быть реализованы, чтобы достичь заданных значений ЦО (компоненты 2-4 ДК).

\section{4. Методика получения данных по многопараметрической оценке ДРВ- проектов}

\section{1. Анкета по многопараметрической оценке проектов ДРВ}

В 2016 - 2018 годах был проведен опрос международного сообщества добровольцев с целью определения взвешенной многопараметрической оценки по проектам ДРВ, в которых добровольцы принимают участие. Многопараметрическая оценка состояла из следующих 9 характеристик проекта ДРВ:

1. Ясный замысел проекта;

2. Научная составляющая проекта;

3. Качество научных и научно-популярных публикаций по теме проекта;

4. Дизайн проекта (сайт, сертификат, screensaver);

5. Информативность материалов на сайте проекта;

6. Визуализация результатов проекта (фото, видео, инфографика);

7. Организация обратной связи (форумы, чаты и др.);

8. Стимулирование участия кранчера в проекте (соревнования, система начисления баллов, призы);

9. Простота присоединения к проекту (нет барьеров и организационных и технических сложностей).

Каждая характеристика имела не только оценку от -2 до 2, но и свой вес от 0 до 10.

В 2016 году были сформированы варианты анкеты на русском и английском языке. В 2018 году были добавлены немецкий и французский вариант анкеты. Всего было собрано 402 анкеты (см. Таблицу 1).

Таблица 1. Распределение анкет по проектам ДРВ

\begin{tabular}{|l|c|}
\hline \multicolumn{1}{|c|}{ Проект ДРВ } & Количество анкет \\
\hline SAT@home & 56 \\
SETI@home & 32 \\
Einstein@home & 29 \\
Gerasim@home & 29 \\
Rosetta@home & 28 \\
Asteroids@home & 21 \\
LHC@home & 20 \\
RakeSearch & 18 \\
MilkyWay@home & 18 \\
PrimeGrid & 18 \\
Folding@home & 14 \\
POGS@home & 14 \\
Collatz Conjecture & 13 \\
World Community Grid & 13 \\
Acoustics@home & 8 \\
Ocтальныe проекты & 71 \\
\hline Bсего & $\mathbf{4 0 2}$ \\
\hline
\end{tabular}


Так как информация об анкете в основном распространялась на русскоязычных сайтах добровольцев, а в преамбуле к анкете 2018 года была рекомендация оценить прежде всего проекты ДРВ российского происхождения, то получилось следующее распределение количества анкет по языкам (см. Таблицу 2).

Таблица 2. Распределение анкет по языкам

\begin{tabular}{|l|c|}
\hline \multicolumn{1}{|c|}{ Проект ДРВ } & Количество анкет \\
\hline Русский & 252 \\
Английский & 133 \\
Французский & 9 \\
Немецкий & 8 \\
\hline
\end{tabular}

\section{2. Вес характеристик}

Для увеличения информативности характеристики были отсортированы по убыванию веса. В этом случае лепестковая диаграмма с усредненными весами по всем проектам будет выглядеть как на Рисунке 1.

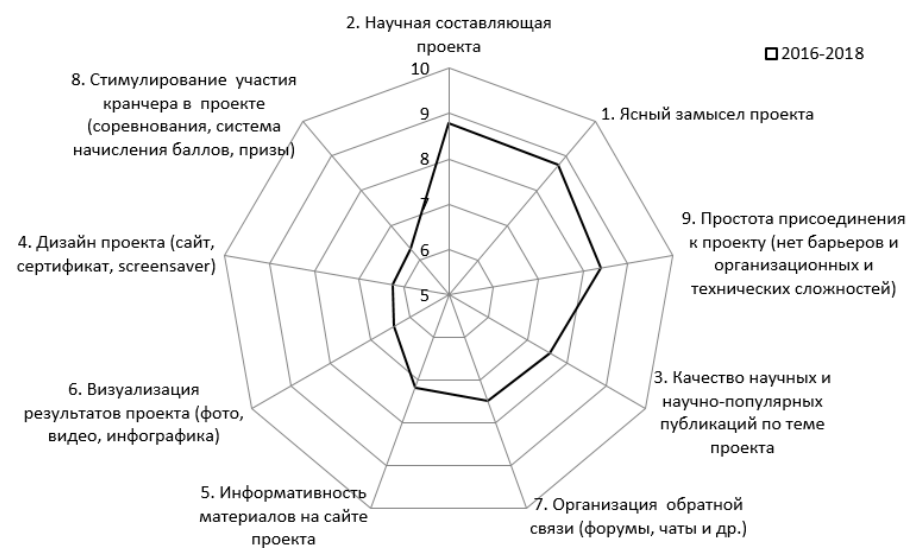

Рис. 1. Усредненные веса характеристик проекта ДРВ

Значения усредненных весов характеристик проектов за период с 2016 по 2018 год изменились незначительно (см. Рисунок 2).

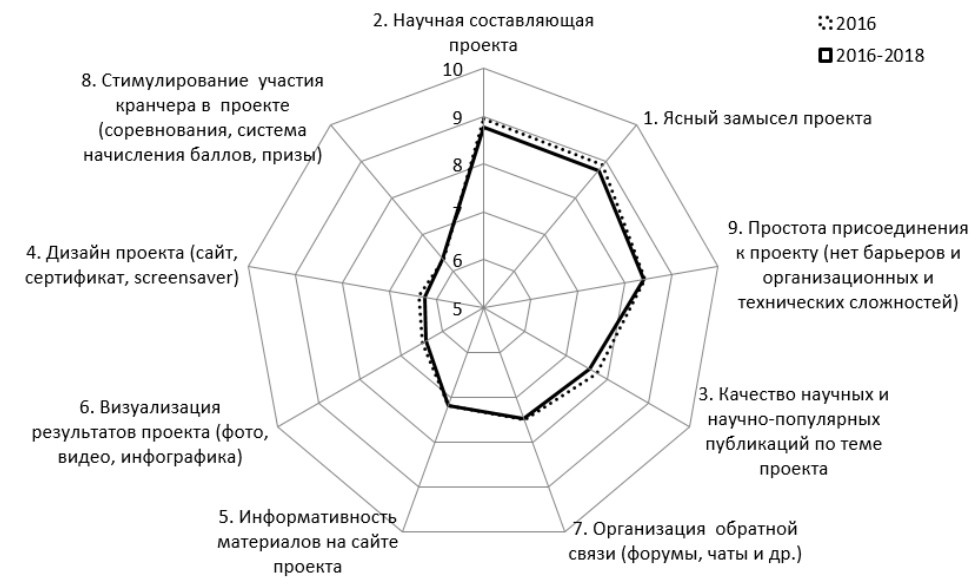

Рис. 2. Изменение усредненных весов за период с 2016 по 2018 годы 
Поэтому в качестве усредненной оценки и усредненных весов будут приниматься усредненные данные в течение всего периода 2016-2018 годов.

\section{3. Многопараметрическая оценка проекта ДРВ Gerasim@home в 2016 году}

При анализе материалов многопараметрической оценки проекта Gerasim@home в 2016 году (рис.3 и таблица 3), установлено, что по пяти из девяти характеристик этот ДРВпроект имел низкие оценки от 3 до 4 баллов по пятибалльной шкале, в том числе:

- качество научных и научно-популярных публикаций по теме проекта - чуть выше 3 баллов (0.06 в нашей шкале от -2 до +2 баллов);

- организация обратной связи (форумы,чаты и др.) - около 3.3 балла (0.35);

- дизайн проекта (сайт, сертификат, screensaver) - чуть выше 3.5 балла (0.53);

- стимулирование участия кранчера в проекте (соревнования, система начисления баллов, призы) - около 3.7 балла (0.76);

- информативность материалов на сайте проекта - 3.9 балла (0.94).

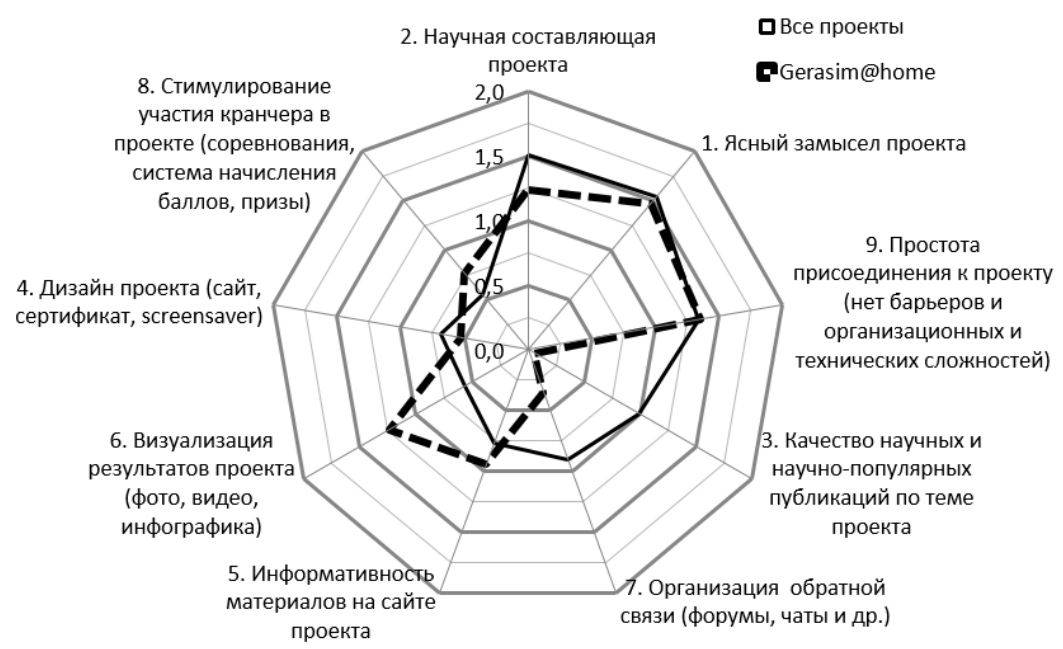

Рис. 3. Лепестковая диаграмма проекта Gerasim@home по анкетам за 2016 год

Таблица 3. Веса и оценки характеристик для проекта Gerasim@home в 2016 году

\begin{tabular}{|c|c|c|c|c|}
\hline № & Характеристики & $\begin{array}{l}\text { Вес для } \\
\text { всех } \\
\text { проектов }\end{array}$ & $\begin{array}{l}\text { Вес для } \\
\text { проекта } \\
\text { Gerasim } \\
\text { ahome }\end{array}$ & $\begin{array}{c}\text { Оценка } \\
\text { характеристик }\end{array}$ \\
\hline 1 & Ясный замысел проекта & 8.89 & 8.94 & 1.47 \\
\hline 2 & Научная составляющая проекта & 8.94 & 8.06 & 1.24 \\
\hline 3 & $\begin{array}{l}\text { Качество научных и научно-популярных } \\
\text { публикаций по теме проекта }\end{array}$ & 7.75 & 6.35 & 0.06 \\
\hline 4 & Дизайн проекта (сайт, сертификат, screensaver) & 6.37 & 7.35 & 0.53 \\
\hline 5 & Информативность материалов на сайте проекта & 7.17 & 6.59 & 0.94 \\
\hline 6 & $\begin{array}{l}\text { Визуализация результатов проекта (фото, видео, } \\
\text { инфографика) }\end{array}$ & 6.49 & 8.71 & 1.24 \\
\hline 7 & Организация обратной связи (форумы, чаты и др.) & 7.49 & 7.18 & 0.35 \\
\hline 8 & $\begin{array}{l}\text { Стимулирование участия кранчера в проекте } \\
\text { (соревнования, система начисления баллов, } \\
\text { призы) }\end{array}$ & 6.31 & 8.00 & 0.76 \\
\hline 9 & $\begin{array}{l}\text { Простота присоединения к проекту (нет барьеров } \\
\text { и организационных и технических сложностей) }\end{array}$ & 8.44 & 8.35 & 1.35 \\
\hline
\end{tabular}


Команда проекта приняла решение предпринять усилия по исправлению положения дел по названным аспектам работы, использовав подход $\mathrm{D}$ к построению и реализации дорожной карты совершенствования своего проекта. При этом значения целевых ориентиров (ЦО) для пяти выбранных характеристик были установлены на значение 1 балл (в шкале от -2 до +2 баллов). Обоснование такого выбора значений ЦО оправдывалось тем, что учитывались возможности команды проекта, с одной стороны, и закладывалась стратегия «довести» значения оценок данных характеристик до уровня оценок для всех проектов (рис.3) параллельно достигая более высоких значений ЯКиндекса для своего проекта.

\section{4. Многопараметрическая оценка проекта ДРВ Gerasim@home в 2018 году}

Почти двухлетняя целенаправленная работа команды проекта Gerasim@home дала свои плоды. По четырем из пяти выбранных характеристик целевые ориентиры были превышены (см. рис.4). Сравнив данные таблиц 3 и 4, мы видим, что лишь по одной характеристике не удалось достичь (незначительно) ЦО - это «качество научных и научно-популярных публикаций по теме проекта». Здесь оценка составила около 3.9 баллов (0.93).

Одновременно, в ходе совершенствования деятельности рассматриваемого проекта, сотрудники задумались о необходимости более четкой формулировки замысла и научной составляющей (см. две наиболее значимые характеристики №2 и №1 на рис.4 и в таблице 4).

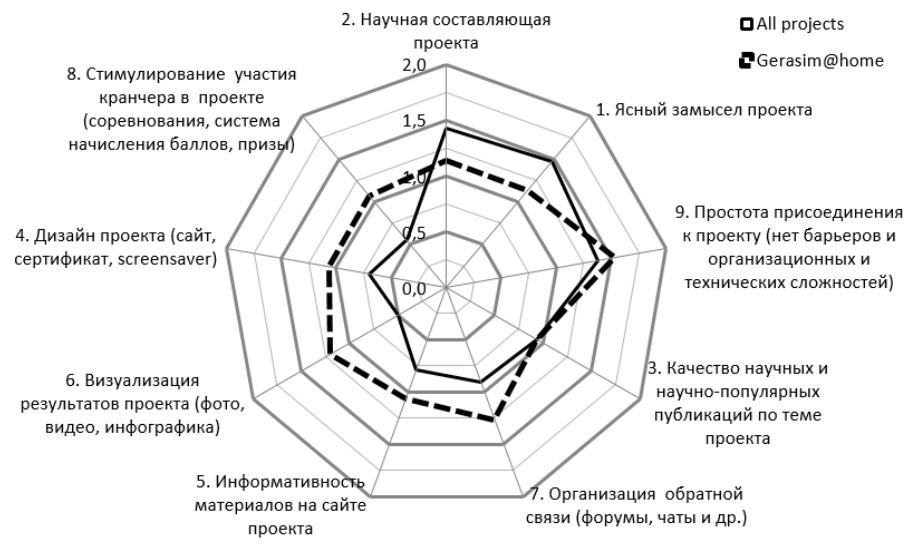

Рис. 4. Лепестковая диаграмма проекта Gerasim@home по анкетам за 2018 год

Команда проекта добровольных распределенных вычислений Gerasim@home на протяжении всего времени работы не располагала достаточными ресурсами для достижения целевых ориентиров по всем представленным характеристикам. При составлении в 2016 году лепестковой диаграммы по многопараметрической оценке проекта Gerasim@home сообществом добровольцев были выявлены 2 характеристики, оценки которых были существенно ниже, чем усредненные оценки по всем проектам (рис. 3). За 2 года (2016-2018) были приложены усилия по улучшению данных показателей. В результате использования подхода $\mathrm{D}$, оценки по данным показателям, улучшились до усредненных по всем проектам (рис.4). Также стоит отметить, что ЯК-индекс проекта Gerasim@home в 2016 году был равен 0.589, а в 2018 году значение этого индекса у проекта Gerasim@home выросло до 0.628. 
Таблица 4. Веса и оценки характеристик для проекта Gerasim@home в 2018 году

\begin{tabular}{|c|c|c|c|c|}
\hline № & Характеристики & $\begin{array}{l}\text { Вес для } \\
\text { всех } \\
\text { проектов }\end{array}$ & $\begin{array}{c}\text { Вес для } \\
\text { проекта } \\
\text { Gerasim } \\
\text { ahome }\end{array}$ & $\begin{array}{c}\text { Оценка } \\
\text { характеристик }\end{array}$ \\
\hline 1 & Ясный замысел проекта & 8.89 & 7.73 & 1.13 \\
\hline 2 & Научная составляющая проекта & 8.94 & 7.73 & 1.13 \\
\hline 3 & $\begin{array}{l}\text { Качество научных и научно-популярных } \\
\text { публикаций по теме проекта }\end{array}$ & 7.75 & 7.60 & 0.93 \\
\hline 4 & Дизайн проекта (сайт, сертификат, screensaver) & 6.37 & 7.60 & 1.07 \\
\hline 5 & Информативность материалов на сайте проекта & 7.17 & 8.07 & 1.07 \\
\hline 6 & $\begin{array}{l}\text { Визуализация результатов проекта (фото, видео, } \\
\text { инфографика) }\end{array}$ & 6.49 & 7.93 & 1.20 \\
\hline 7 & $\begin{array}{l}\text { Организация обратной связи (форумы, чаты и } \\
\text { др.) }\end{array}$ & 7.49 & 8.53 & 1.27 \\
\hline 8 & $\begin{array}{l}\text { Стимулирование участия кранчера в проекте } \\
\text { (соревнования, система начисления баллов, } \\
\text { призы) }\end{array}$ & 6.31 & 7.53 & 1.07 \\
\hline 9 & $\begin{array}{l}\text { Простота присоединения к } \\
\text { барьеров и п ороекту } \text { (нет } \\
\text { сложностей) }\end{array}$ & 8.44 & 8.53 & 1.53 \\
\hline
\end{tabular}

\section{Заключение}

Предложенные подходы по улучшению проекта ДРВ для сообщества добровольцев позволяют команде организаторов минимизировать усилия по сопровождению проектов ДРВ и сконцентрироваться на наиболее важных для них целевых ориентирах. Собранные данные в рамках опроса по оценке проектов ДРВ позволяют не только оценить динамику многопараметрической оценки проекта ДРВ, но и сравнить текущие показатели проекта с другими проектами ДРВ. Предложенные подходы можно применять не только в проектах ДРВ, но и в других проектах, в которых необходимо взаимодействовать с сообществом добровольцев.

Работа выполнена при финансовой поддержке РФФИ в рамках научных проектов №1907-00802, 18-57-06003.

\section{Литература}

[1] Benoit, et al. Scheduling Concurrent Bag-of-Tasks Applications on Heterogeneous Platforms // IEEE Trans. Computers. 2010. Vol. 59, № 2. P. 202-217.

[2] Anderson D.P. "BOINC: a system for public-resource computing and storage", Grid Computing, IEEE, 2004 Proceedings. Fifth IEEE/ACM International Workshop. DOI: 10.1109/GRID.2004.14.

[3] The server of statistics of voluntary distributed computing projects on the BOINC platform. URL: http://boincstats.com (date of access: 15.02.2019).

[4] Site of World Community Grid project. URL: http://www.worldcommunitygrid.org. (date of access: 24.02.2019).

[5] Kurochkin I. I. The umbrella project of volunteer distributed computing Optima@ home // CEUR Workshop Proceedings. 2017. Vol. 1973. P. 35-42.

[6] Posypkin M., Semenov A., Zaikin O. Using BOINC desktop grid to solve large scale SAT problems // Computer Science. 2012. Vol. 13 (1). P. 25-34. 
[7] Simpson R., Page K.R., De Roure D. Zooniverse: observing the world's largest citizen science platform // Proceedings of the 23rd international conference on world wide web. ACM, 2014. P. 1049-1054.

[8] Bonney R. et al. Citizen science: a developing tool for expanding science knowledge and scientific literacy // BioScience. 2009. Vol. 59 (11). P. 977-984.

[9] Wynn J. Citizen science in the digital age: rhetoric, science, and public engagement. University of Alabama Press, 2017.

[10]Jennett C. et al. Motivations, learning and creativity in online citizen science // Journal of Science Communication. 2016. Vol. 15 (3).

[11]Карасёв О.И., Дорошенко М.Е. Использование метода дорожных карт для формирования перспективного видения развития мегарегулирования финансового сектора в России // Вестн. Моск. ун-та. Сер. 6. Экономика. 2015. № 4. С.75-98.

[12]Матич Л. Ю. Системный подход при разработке дорожных карт для российских отраслей и компаний // Труды ИСА РАН. 2016. Т. 66, № 3. С. 86-97.

[13]Ковалев В.И. Дорожная карта инновационного развития предприятия // Universum: экономика и юриспруденция. 2014.2 № $4 . \quad$ URL: http://cyberleninka.ru/article/n/dorozhnaya-karta-innovatsionnogo-razvitiyapredpriyatiya.

[14]Белоусов Д.Р., Сухарева И.О., Фролов А.С. Метод «картирования технологий» в поисковых прогнозах // Форсайт. 2012. Т. 6, № 2. С. 6-16.

[15]Черепанов М.А. Дорожная карта как инструмент управления развитием организации // Проблемы управления. 2014. Вып. 5 (30).

[16]Технология дорожного картирования. URL: http://www.unido.org/fileadmin/import/ 16963_TechnologyRoadmapping.pdf (дата обращения 23.02.2019).

[17]Манчулянцев О.А., Павлычева Е.Ю., Краузова Е.Н., Ткачева А.В. Дорожные карты российского бизнеса: Аналитический отчет по исследованию. М.: Open Innovation Inc., 2012. $64 \mathrm{c}$.

[18] Гохберг Л.М. Будущее как стратегическая задача // Форсайт. 2007. № 1 (1).

[19]Willyard C.H., McClees C.W. Motorola's technology roadmap process // Research Management. 1987. Р. 13-19.

[20]Якимец В.Н., Курочкин И.И. Добровольные распределенные вычисления в России: социологический анализ // Информационное общество: образование, наука, культура и технологии будущего Труды XVIII объединенной конференции «Интернет и современное общество» (IMS-2015). СПб, 2015. С. 345-352.

[21] Yakimets V.N., Kurochkin I.I. Analysis of results of the rating of volunteer distributed computing projects // Russian Supercomputing Days 2018, September 24-25, 2018, Moscow, Russia: Proceedings of international conference, MSU, 2018, P.893-908.

\title{
Roadmap for Improving Volunteer Distributed Computing Project Performance Based on Multi-Parameter Assessments
}

\author{
V.N. Yakimets ${ }^{1,2}$ and I.I. Kurochkin ${ }^{1}$ \\ ${ }^{1}$ Institute for Information Transmission Problems of Russian Academy of Sciences \\ 2 The Russian Presidential Academy of National Economy and Public Administration \\ iakimets@mail.ru, kurochkin@iitp.ru
}

The methodology for improving activities of distributed computing projects with the participation of volunteers based on the results of multiparameter and index estimates is considered. Such project estimates provide a strategy for improving the work of a voluntary distributed computing project to streamline the process of building the appropriate road map. The concept of "target reference point" is introduced, with the help of which the project team, taking into account its specificity and available resource capabilities, in all parameters forms a vector of 
target values that are going to be achieved within a given time. Four options for selecting "targets" that set the direction of the roadmap for the development of the project of voluntary distributed computing are proposed. The results of a survey of the volunteer community on multiparameter evaluation of projects for 2016-2018 are described. The example of the Gerasim@home project shows how to improve its activities for the selected version of the targets. The results of changes in the project activities are discussed.

Keywords: distributed computing, voluntary distributed computing project, VDC project, desktop grid, BOINC, multi-parameter assessments of the project

Reference for citation: Yakimets V.N., Kurochkin I.I. Roadmap for Improving Volunteer Distributed Computing Project Performance Based on Multi-Parameter Assessments // Information Society: Education, Science, Culture and Technologies of the Future. Vol. 3 (Proceedings of the XXII International Joint Scientific Conference «Internet and Modern Society», IMS-2019, St. Petersburg, June 19-22, 2019). - St. Petersburg: ITMO University, 2019. P. 41 - 52. DOI: 10.17586/2587-8557-2019-3-41-52

\section{Reference}

[1] Benoit, et al. Scheduling Concurrent Bag-of-Tasks Applications on Heterogeneous Platforms // IEEE Trans. Computers. 2010. Vol. 59, № 2. P. 202-217.

[2] Anderson D.P. "BOINC: a system for public-resource computing and storage", Grid Computing, IEEE, 2004 Proceedings. Fifth IEEE/ACM International Workshop. DOI: 10.1109/GRID.2004.14.

[3] The server of statistics of voluntary distributed computing projects on the BOINC platform. URL: http://boincstats.com (date of access: 15.02.2019).

[4] Site of World Community Grid project. URL: http://www.worldcommunitygrid.org. (date of access: 24.02.2019).

[5] Kurochkin I. I. The umbrella project of volunteer distributed computing Optima@ home // CEUR Workshop Proceedings. 2017. Vol. 1973. P. 35-42.

[6] Posypkin M., Semenov A., Zaikin O. Using BOINC desktop grid to solve large scale SAT problems // Computer Science. 2012. Vol. 13 (1). P. 25-34.

[7] Simpson R., Page K.R., De Roure D. Zooniverse: observing the world's largest citizen science platform // Proceedings of the 23rd international conference on world wide web. ACM, 2014. P. 1049-1054.

[8] Bonney R. et al. Citizen science: a developing tool for expanding science knowledge and scientific literacy // BioScience. 2009. Vol. 59 (11). P. 977-984.

[9] Wynn J. Citizen science in the digital age: rhetoric, science, and public engagement. University of Alabama Press, 2017.

[10]Jennett C. et al. Motivations, learning and creativity in online citizen science // Journal of Science Communication. 2016. Vol. 15 (3).

[11]Karasyov O.I., Doroshenko M.E. Ispol'zovanie metoda dorozhnyh kart dlya formirovaniya perspektivnogo videniya razvitiya megaregulirovaniya finansovogo sektora v Rossii // Vestn. Mosk. un-ta. Ser. 6. Ekonomika. 2015. № 4. P.75-98.

[12]Matich L. YU. Sistemnyj podhod pri razrabotke dorozhnyh kart dlya rossijskih otraslej i kompanij // Trudy ISA RAN. 2016. T. 66, № 3. P. 86-97.

[13]Kovalev V.I. Dorozhnaya karta innovacionnogo razvitiya predpriyatiya // Universum: ekonomika i yurisprudenciya. 2014. № 4. URL: http://cyberleninka.ru/article/n/dorozhnayakarta-innovatsionnogo-razvitiyapredpriyatiya.

[14]Belousov D.R., Suhareva I.O., Frolov A.S. Metod «kartirovaniya tekhnologij» v poiskovyh prognozah // Forsajt. 2012. T. 6, № 2. P. 6-16.

[15]Cherepanov M.A. Dorozhnaya karta kak instrument upravleniya razvitiem organizacii // Problemy upravleniya. 2014. Vol. 5 (30). 
[16]Tekhnologiya dorozhnogo kartirovaniya. URL: http://www.unido.org/fileadmin/import/ [17]16963_TechnologyRoadmapping.pdf (data obrashcheniya 23.02.2019).

[18]Manchulyancev O.A., Pavlycheva E.YU., Krauzova E.N., Tkacheva A.V. Dorozhnye karty rossijskogo biznesa: Analiticheskij otchet po issledovaniyu. M.: Open Innovation Inc., 2012. $64 \mathrm{p}$.

[19]Gohberg L.M. Budushchee kak strategicheskaya zadacha // Forsajt. 2007. № 1 (1).

[20]Willyard C.H., McClees C.W. Motorola's technology roadmap process // Research Management. 1987. P. 13-19.

[21] Yakimets V.N., Kurochkin I.I. Voluntary distributed computing in Russia: a sociological analysis. Collection of scientific articles of the XVIII Joint Conference "Internet and Contemporary Society" (IMS-2015), St. Petersburg, June 23, 2015, St. Petersburg: ITMO University, 2015. Sc.345-352. ISBN 978-5-7577-0502-6.

[22] Yakimets V.N., Kurochkin I.I. Analysis of results of the rating of volunteer distributed computing projects // Russian Supercomputing Days 2018, September 24-25, 2018, Moscow, Russia: Proceedings of international conference, MSU, 2018, pp.893-908. 